\title{
Universitäre Ärztebildung
}

Peter Spinnler

Dr. med. Peter Spinnler

Rütistrasse 2

CH-5400 Baden

Tel. 0562229982

pesp@bluewin.ch

spinnler.val.muestair@bluewin.ch
Momentan wird eine Verkürzung des Medizinstudiums durch frühzeitige Spezialisierung auf Kosten von breiter, medizinischer Ausbildung zur Diskussion gestellt. Die Debatte zu diesem Thema ist wichtig. Eine frühzeitige Spezialisierung für Medizinstudenten ist kritisch zu hinterfragen. Die Diskussion zu dieser Thematik muss auch grundsätzliche Überlegungen zum Thema «Spezialisierung» einschliessen.

Ohne Zweifel haben Wissen und Machbarkeit in der Medizin in den letzten 50 Jahren enorm zugenommen und werden weiter zunehmen. Diese Entwicklung hat bei der gleichbleibenden Grösse der menschlichen Köpfe und der Lebensdauer unvermeidlich eine Spezialisierung und Subspezialisierung zur Folge, also werden Ärzte quasi zwingend zu (Super-)Spezialisten. Zu Nachteilen jedes Spezialistentums hat Nietzsche 1886 geschrieben: «An dem Buche eines Gelehrten ist fast immer auch etwas Drückendes, Gedrücktes: der «Spezialist kommt irgendwo zum Vorschein, sein Eifer, sein Ernst, sein Ingrimm, seine Überschätzung des Winkels, in dem er sitzt und spinnt, sein Buckel - jeder Specialist hat seinen Buckel» und weiter: «Jede Art Meisterschaft zahlt sich theuer auf Erden, wo vielleicht Alles sich zu theuer zahlt: man ist Mann seines Fachs um den Preis, auch das Opfer seines Fachs zu sein.» («Die fröhliche Wissenschaft», 5. Buch, Ziff. 366: «Angesichts eines gelehrten Buches».)

Einerseits wird eine frühzeitige Spezialisierung zum Zwecke einer Verkürzung des Medizinstudiums aus ökonomischen Gründen gefordert: Studenten sollen nicht mit Wissen beladen und belastet werden, das sie ohnehin nach dem Staatsexamen nie mehr brauchen, angeblich weil es «nutzlos» ist. Andererseits: In urbanen Bereichen melden sich Patienten bei Erkrankung meist direkt bei dem Spezialisten, den sie für ihr Anliegen als den richtigen erachten. Dies wird als Argument gegen die Managed-Care-Modelle ins Feld geführt: Patienten bevorzugen den eigenständig gewählten Spezialisten. Doch merken diese Kranken oft nicht oder erst im nachhinein, dass sie damit bei einem «Nur-Spezialisten» landen können.

Mehrheitlich streben Medizinstudenten nach dem Staatsexamen eine Facharztausbildung an, offensichtlich weil der Berufsalltag des Allgemeinpraktikers als mühsam und wenig interessant beurteilt wird: Der Allgemeinpraktiker - so

\section{Formation universitaire}

\section{des médecins}

La teneur du savoir enseigné doit être adaptée à la capacité de réception d'un bon étudiant. Il est souhaitable dès lors et peut-être obligatoire que l'étudiant en médecine choisisse sa future spécialisation pendant ses études déjà.

Cependant, il est essentiel de prévenir tout dommage en permettant, pendant les études et pour toutes les «directions spéciales», la formation d'une «compétence de l'esprit». L'étudiant à la fin de ses études de base (bachelor) doit disposer d'un savoir spécialisé qui s'accompagne déjà d'une expérience médicale du savoir enseigné auprès du patient, fondée sur des exemples qui lui montrent le «comment» de ce savoir. L'acquisition de ces compétences par l'expérience demande du temps, et ce temps ne saurait être sacrifié à l'exigence légitime d'un raccourcissement des études. Brièvement dit: l'étude spécialisée peut demander de plus petites quantités de matière enseignée. Mais il s'agit aussi de prôner dans le même temps l'apprentissage intensif de la compétence diagnostique et thérapeutique, consistant à synthétiser I'individuel en un tout: le sujet avec un organe malade doit toujours être abordé, vu et pensé comme un «être humain complet».

L'homme et la femme de notre environnement culturel, grâce au savoir et aux possibilités accrues de la médecine, peuvent vivre plus longtemps et supporter des maladies chroniques plus confortablement qu'autrefois. On doit néanmoins se poser la question de savoir si nous bénéficions d'une meilleure qualité de vie ou si nous sommes plus heureux que nos ancêtres? Une vie plus longue est-elle une «meilleure vie»? Bref, l'amélioration de notre savoir et les prouesses techniques de la médecine d'aujourd'hui sont-ils appropriés à notre condition d'être humain? 
etwa die Meinung dieser jungen Ärzte - kann nur noch Patientenwege verwalten, eigenverantwortlich und in eigener Regie leitet er relativ selten Diagnostik und Therapie. Zudem kann ein Superspezialist seinen Arbeitsalltag sehr gut strukturieren: Dieser Mediziner zwingt den Patienten seine Verfügbarkeit auf. Anders der Allgemeinpraktiker und Hausarzt, der sich nach den Patienten und deren Notfallsituation zu richten hat, allenfalls auch einspringen muss, wenn ein Zugang zum Spezialisten nicht oder erst nach längerer Wartezeit möglich ist.

\section{Das Ganze und seine Teile}

Einmal zeigt sich hier eine Problematik, die nicht allein das Spezialistentum in der Medizin betrifft, vielmehr in manchen Bereichen unserer Gesellschaft eine Rolle spielt. Die einzelnen Spezialisten reden zunehmend je eigene Sprachen und denken ihrem Spezialsystem entsprechend und so wird ein Kommunizieren zwischen verschiedenen Spezialisten erschwert oder manchmal gar getrübt: Sie verstehen andere oft kaum, denn sie sind in ihrem Spezialistendenken gefangen. Erinnert dies nicht an die Sprachverwirrung beim Turmbau zu Babylon? Wir werden bei extremer Spezialisierung Gefangene des Details, eben «Mann und Opfer des Fachs». Je mehr wir unser Wissen vermehren, um so mehr droht eine Zersplitterung. Das Ganze geht dabei irgendwie verloren, und dies kann zu einem Sinnverlust führen.

Dann sind wir daran - unsere Gesellschaft scheint dies bisher in Kauf zu nehmen -, nach den Zähnen beim Zahnarzt weitere Organe bei je zuständigen Spezialärzten abzugeben. Patienten glauben dadurch, direkten Zugang zur besten und modernsten Diagnostik und Therapie gewinnen zu können.

Da taucht plötzlich die Frage auf: Wo ist Platz statt für den Träger der Organe für das Individuum, für mein Ich? Ein «over-all», der ganze Mensch, der mehr ist als ein Zusammenspiel seiner Teile, wird zunehmend vernachlässigt. Jedes Ganze ist mehr als seine Teile, auch bei Maschinen. Besonders gross aber ist dieses Mehr beim «ganzen Menschen». Das «over-all» in der Medizin droht als Folge der Superspezialisierung zu verschwinden, weil es die auf ein Organ beschränkte Sicht komplizieren, ja gar stören könnte, weil die Zeit für ein Eingehen auf das Ganze fehlt, weil die äusseren Möglichkeiten des Spezialisten oft überstiegen würden.

Wir sind uns wohl einig darüber, dass «mein Arzt» kein oder nicht nur ein Organtechniker sein darf. Ich trete meinem Arzt nicht als krankes Organ, als Objekt gegenüber, vielmehr als ein anderes, Rat oder Hilfe suchendes Subjekt, als kranker Mensch. Klar, der Arzt muss im Einzelfall Zugang zu sehr spezialisiertem Wissen und den notwendigen technischen Fertigkeiten haben: Dieses Können ist Voraussetzung für eine zeitgemässe und gute Behandlung eines Patienten. Dazu gehört auch die berechtigte Überweisung eines Patienten mit einer klar umschriebenen Fragestellung an den zuständigen Spezialisten; in diesen Situationen wird dieser Nur-Spezialist zum Helfer «meines Arztes». Hüten wir uns vor der Sicht, die Probleme einer Superspezialisierung könnten allein mit modernen Mitteln, z.B. Telemedizin, gelöst werden. Auch ein Netzwerk von Ärzten kann die Begegnung mit dem «Arzt des Ganzen» niemals ersetzen.

Allerdings, auch ein Spezialarzt kann ein guter Arzt sein, er kann «mein Arzt» sein und mein Vertrauen geniessen. Dafür ist von zentraler Bedeutung, dass er über Wissen und Fertigkeiten hinaus eines besitzt: Kompetenz im Umgang zwischen Menschen und im ärztlichen Umgang mit seinem Wissen (und seinem Nichtwissen): Wie geht der Arzt ein Problem an? Wie verschafft er sich ihm aktuell fehlendes Wissen? Wie pflegt er die Beziehung zweier Subjekte neben einer sachlichen und möglichst objektiven Beurteilung und Behandlung einer Organstörung, demnach die Begegnung mit dem «ganzen Menschen»? Wie schafft er eine für den Patienten annehmbare zeitliche Verfügbarkeit? Wie stark bemüht er sich um eine patientengerechte Information des Kranken? Oder nochmals anders: Wie steht es um die Kultur des Zuhörens, des Gesprächs, um die Kultur der Begegnung eines Arztes mit seinen Patienten?

\section{(Aus-)Bildung von Ärzten}

Wie bildet «man» solche Ärzte? Die Vermittlung von Wissen und technischen Fertigkeiten steht im Medizinstudium im Vordergrund. Wissen und technische Fertigkeiten sind aber schlicht nur Voraussetzungen für ärztliches Tun, sie sind relativ einfach weiterzugeben, zu dozieren, zu erklären, in Lehrbüchern nachzulesen; sie sind gut erlernbar, sie sind gut prüfbar. Ein Einsatz moderner Unterrichtsmittel ist wesentlich, aber dadurch kann schwerlich eine Kompetenz im zwischenmenschlichen Umgang zwischen einem, der leidet und dadurch abhängig geworden ist, und einem, der hilfreich mit Wissen und technischen Fertigkeiten beistehen kann, vermittelt werden. Diese Kompetenz ist schwer zu erlernen und sehr schwer zu lehren. Es stellt sich die Frage, ob dafür genügend geeignete Lehrer-Vorbilder gefunden werden. Neue gesetzliche Regelungen 
mit Betonung der sozialen Kompetenzen und dem frühzeitigen Patientenkontakt allein genügen in der Ärzteausbildung nicht. Vielmehr ist die Entwicklung einer neuen Haltung bei Gesellschaft, Lehrenden und Studierenden anzustreben.

Besonders schwierig wird sich die Ausbildung gestalten für zukünftige Hausärzte und für Ärzte, die später in abgelegenen, ländlichen Gebieten arbeiten. Die Allgemeinpraktiker müssen gut ausgebildet werden in der Umsetzung von Wissen, in der Fähigkeit, komplexe Situationen auf Einfaches zurückzuführen, in der Führung von Gesprächen; sie müssen, allenfalls zusammen mit Spezialisten, den Kranken hinführen zum Entscheiden. Ein Hausarzt muss Selbstvertrauen, Selbstkritik und Berufsstolz pflegen, so dass er erfolgreich Spezialisten beiziehen und als seine Helfer einsetzen kann. - Analoges gilt für die Ärzte, die in abgelegenen Gebieten arbeiten; sie müssen viel wissen und vieles selbst praktisch anwenden und leisten können. Für sie ist wichtig, sich kurzfristig fehlendes Wissen zu beschaffen und situationsgerecht einzusetzen (evtl. unterstützt mittels Telemedizin). Sie müssen vor allem die eigenen Grenzen kennen und wieder und wieder überprüfen und sich hinterfragen.

Die Kompetenz im «wissenden Umgang» mit Kranken jedoch lässt sich wohl nur im Erleben von Beispielhaftem - anhand einer vorgeführten Kompetenz - erfahren. Solches Erfahren, solches Erleben schulen wesentlich die Fähigkeiten eines Studenten oder jungen Arztes, Wissen sinnvoll einsetzen und damit einem kranken Menschen hilfreich begegnen zu können. Wenn ein Student das Glück hat, zu erleben und zu erfahren, wie ein Lehrer solches gekonnt vorlebt, so kann er - wenn er einer universitären Bildung würdig ist - analog vorzugehen sich bemühen. Die Menge des Wissens und der technischen Fertigkeiten ist dabei von zweitrangiger Bedeutung, denn der ärztliche Umgang mit Wissen und den technischen Fertigkeiten bei einem Patienten ist nicht eine Frage der Quantität, vielmehr eine solche der Qualität. Fachliche Zuständigkeit, es sei wiederholt, ist Voraussetzung, nur Voraussetzung. Dann aber, zentral, geht es um das «wie» und nicht mehr lediglich um ein «was».

Die Kompetenz zur Sicht des Ganzen muss mühsam herangebildet werden. Denn es ist diese Kompetenz, die hohe Bildung - Universitas auszeichnet: die Beziehung Ganzes-Einzelnes immer wieder neu zu erkunden und zu vertiefen. Friederich Schiller hat sich 1789 in seiner An- trittsvorlesung «Was heisst und zu welchem Ende studiert man Universalgeschichte?» zu dieser Thematik sehr pointiert geäussert. Er konfrontiert den «Brotgelehrten» mit dem philosophischen Kopf, etwa wenn er schreibt: «Nicht was er treibt, sondern wie er das, was er treibt, behandelt, unterscheidet den philosophischen Geist. Wo er auch stehe und wirke, er steht immer im Mittelpunkt des Ganzen; und soweit ihn auch das Objekt seines Wirkens von seinen übrigen Brüdern entferne, er ist ihnen verwandt und nahe durch einen harmonisch wirkenden Verstand, er begegnet ihnen, wo alle hellen Köpfe einander finden.»

\section{Zusammenfassung}

Die Menge des vermittelten Wissens muss der Aufnahmekapazität eines guten Studenten angemessen sein; es ist demnach wünschbar, vielleicht sogar zwingend, dass für Medizinstudenten schon während der Studienzeit eine Spezialisierung erfolgt.

Jedoch: Dies kann nur dann ohne Schaden geschehen, wenn für alle «Spezialrichtungen» während des Studiums der Bildung einer Kompetenz des Geistes zentrale Bedeutung zugestanden wird. Ein Student muss bei Abschluss seines Grundstudiums (Bachelor) über ein spezialisiertes Grundwissen verfügen, darüber hinaus aber schon den ärztlichen Umgang mit dem erworbenen Wissen bei Kranken erlebt und erfahren haben durch Beispiele, die ihm ein «wie» aufzeigen. Die Aneignung solcher Kompetenzen durch Erfahrung braucht Zeit, und diese Zeit darf der berechtigten Forderung nach Studienverkürzung nicht geopfert werden. Auf eine kurze Formel gebracht: Kleinere Stoffmenge an Spezialwissen. Aber gleichzeitig intensivierte Schulung einer diagnostischen und therapeutischen Kompetenz, Einzelnes zu einem Ganzen zu synthetisieren: Das Subjekt mit einem erkrankten Organ ist immer auch als «Ganzer Mensch» zu sehen, zu spüren und zu denken.

Die Menschen in unserem Kulturkreis haben dank dem Zuwachs an Wissen und Machbarkeit in der Medizin eine höhere Lebenserwartung und chronische Leiden können besser gemildert werden als früher, doch es bleibt zu fragen: Sind wir qualitativ gesünder oder gar glücklicher geworden als unsere Vorfahren? Ist ein langes Leben ein «besseres» Leben als ein kürzeres? Kurz, was haben uns Wissen und technische Machbarkeit, die die moderne Medizin zur Verfügung stellt, für unser Menschsein gebracht? 